\title{
Evidence for coupling between charge density waves and phonons in two-dimensional rare-earth tritellurides
}

\author{
M. Lavagnini, ${ }^{1}$ M. Baldini, ${ }^{2}$ A. Sacchetti, ${ }^{1}$ D. Di Castro, ${ }^{2}$ B. Delley, ${ }^{3}$ R. Monnier, ${ }^{1}$ J.-H. Chu, ${ }^{4}$ N. Ru, ${ }^{4}$ I. R. Fisher ${ }^{4}$ \\ P. Postorino, ${ }^{2}$ and L. Degiorgi ${ }^{1}$ \\ ${ }^{1}$ Laboratorium für Festkörperphysik, ETH-Zürich, CH-8093 Zürich, Switzerland \\ ${ }^{2}$ Dipartimento di Fisica and CNR-INFM-Coherentia, Università "La Sapienza," P.le A. Moro 5, I-00185 Rome, Italy \\ ${ }^{3}$ Paul Scherrer Institute, CH-5232 Villigen PSI, Switzerland \\ ${ }^{4}$ Geballe Laboratory for Advanced Materials and Department of Applied Physics, Stanford University, \\ Stanford, California 94305-4045, USA
}

(Received 9 October 2008; published 19 November 2008)

\begin{abstract}
We report on a Raman-scattering investigation of the charge density wave (CDW), quasi-two-dimensional rare-earth tritellurides $R \mathrm{Te}_{3}\left(R=\mathrm{La}, \mathrm{Ce}, \mathrm{Pr}, \mathrm{Nd}, \mathrm{Sm}, \mathrm{Gd}\right.$, and Dy) at ambient pressure, and of $\mathrm{LaTe}_{3}$ and $\mathrm{CeTe}_{3}$ under externally applied pressure. The observed phonon peaks can be ascribed to the Raman-active modes for both the undistorted and the distorted lattices in the CDW state by means of a first-principles calculation. The latter also predicts the Kohn anomaly in the phonon dispersion, driving the CDW transition. The integrated intensity of the two most prominent modes scales as a characteristic power of the CDW-gap amplitude upon compressing the lattice, which provides clear evidence for the tight coupling between the CDW condensate and the vibrational modes.
\end{abstract}

DOI: 10.1103/PhysRevB.78.201101

PACS number(s): 71.45.Lr, 62.50.-p, 63.20.dk, 78.30.-j

The electron-phonon coupling is of fundamental relevance for the development of several types of charge ordering in solids, of which the charge density wave (CDW) state, first predicted by Peierls, ${ }^{1}$ is an interesting realization. Peierls ${ }^{1}$ argued that one-dimensional (1D) metals are intrinsically unstable, and that a new broken-symmetry ground state results from the self-consistent rearrangement of the electronic charge density in response to the (static) modulation of the ionic positions. ${ }^{1,2}$ The new lattice periodicity leads moreover to the opening of a gap at the Fermi level. The consequences of this intimate connection between electronic properties and lattice dynamics have been intensively investigated in a number of prototype quasi-1D materials. ${ }^{3}$

CDWs have been observed in transition-metal dichalcogenides and trichalcogenides, ${ }^{4,5}$ in the ladder compounds $\mathrm{Sr}_{14-x} \mathrm{Ca}_{x} \mathrm{Cu}_{24} \mathrm{O}_{41}{ }^{6},{ }^{6}$ and in some copper oxide hightemperature superconductors ${ }^{7}$ (where they are known as "stripes") as well, suggesting that similar effects are to be expected also in layered quasi-2D systems. A recent theoretical study ${ }^{8}$ confirms that this is indeed the case, and that two orthogonal CDWs may even combine to generate a checkerboardlike charge pattern. However, high-temperature superconductors are bad candidates for a systematic study of the interplay between electronic and phononic degrees of freedom in quasi-2D materials, given the strongly correlated nature of the electrons in these systems. One class of quasi-2D compounds well suited to address this issue are the rare-earth $(R)$ tritellurides. $^{9-11}$ They host a CDW state already at $300 \mathrm{~K}$ and their structure consists of alternating double $\mathrm{Te}(a c)$ planes (where the CDW resides) sandwiched between $R \mathrm{Te}$ layer blocks and stacked along the long $b$ axis in the weakly orthorhombic (pseudotetragonal) cell. In our first optical investigations, we have established the excitation across the CDW gap and discovered that this gap is progressively reduced upon compressing the lattice either with chemical substitution (i.e., by changing $R$ ) or with externally applied pressure. $^{12,13}$
The formation of the CDW condensate in $R \mathrm{Te}_{3}$ only partially gaps the Fermi surface ${ }^{14}$ and therefore these materials remain metallic even well below the critical temperature $T_{\mathrm{CDW}}$ at which the CDW appears. ${ }^{11}$ This prevents the investigation of the phonon modes and more generally of the impact of the lattice dynamics on the CDW state $^{2}$ in an infrared-absorption experiment, as the corresponding signals are overwhelmed by the metallic contribution. We therefore address the issue of the coupling between vibrational modes and CDW condensate in these prototype 2D systems from the perspective of the Raman-scattering response. Our data, combined with the measured CDW gap, and supported by first-principles calculations, allow us to identify the expected Raman-active modes and to determine their evolution under pressure. ${ }^{15}$ We provide clear-cut evidence for a tight coupling between CDW condensate and vibrational modes as well as robust predictions for the incipient Kohn anomaly, i.e., the "freezing in" of a lattice distortion associated with the formation of the CDW phase. $^{1-3}$

Our Raman-scattering experiments were performed on the rare-earth series $R \mathrm{Te}_{3}[R=\mathrm{La}, \mathrm{Ce}, \mathrm{Pr}, \mathrm{Nd}, \mathrm{Sm}, \mathrm{Gd}$, and $\mathrm{Dy}$, with $T_{\mathrm{CDW}}>300 \mathrm{~K}$ (Ref. 11)] at ambient pressure as well as on $\mathrm{LaTe}_{3}$ and $\mathrm{CeTe}_{3}$ under externally applied pressure. The single crystalline samples of $R \mathrm{Te}_{3}$ were grown by slow cooling of a binary melt. ${ }^{10}$ Raman spectra were collected on cleaved [010] surfaces with a commercial micro-Raman spectrometer equipped with a He-Ne laser (632.8 nm wavelength and $16 \mathrm{~mW}$ power), a $20 \times$ microscope objective (10 $\mu^{2}{ }^{2}$ laser spot), a notch filter to reject the elastic contribution, an 1800 lines/mm grating, and a cooled chargecoupled-device detector. Additionally we have performed polarization-dependent Raman experiments on $\mathrm{LaTe}_{3}$ at ambient pressure by varying the angle between the incident light polarization and the crystal axes by means of a $\lambda / 2$ polarization rotator and selecting the scattered polarization parallel to the incident one. High pressures were generated 


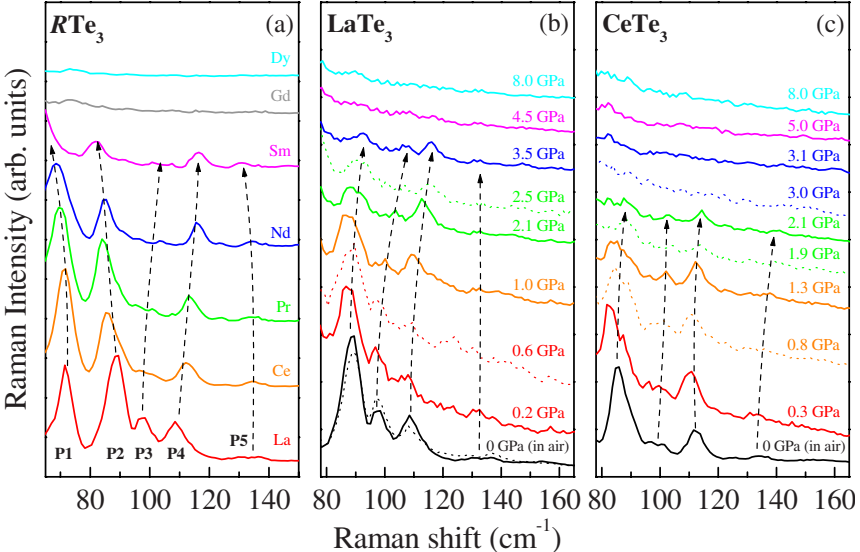

FIG. 1. (Color online) Raman-scattering spectra at $300 \mathrm{~K}$ (a) for the $R \mathrm{Te}_{3}$ series at ambient pressure, and (b) for $\mathrm{LaTe}_{3}$ and (c) $\mathrm{CeTe}_{3}$ for increasing (continuous lines) and decreasing (dashed lines) pressures. All spectra have been shifted for clarity.

by means of a Betsa membrane diamond-anvil cell (DAC) equipped with high-quality type IIA diamonds $(800 \mu \mathrm{m} \mathrm{cu}-$ let diameter) and a stainless-steel gasket $(300 \mu \mathrm{m}$ hole diameter and $50 \mu \mathrm{m}$ thickness $)$. A small $\left(\approx 100 \times 100 \mu \mathrm{m}^{2}\right)$ sample piece was placed inside the DAC together with the pressure-transmitting medium (a 4:1 methanol-ethanol mixture ${ }^{16}$ ) and a small ruby chip for pressure measurement. ${ }^{17}$

Figure 1(a) summarizes the Raman-scattering spectra, collected for the whole $R \mathrm{Te}_{3}$ series (i.e., chemical pressure). Four distinct modes at 72, 88, 98, and $109 \mathrm{~cm}^{-1}$ and a weak bump at $136 \mathrm{~cm}^{-1}$ (labeled $P 1-P 5$, respectively) can be identified in the La compound. The $P 1$ mode slightly softens from $\mathrm{La}$ to $\mathrm{Nd}$ and slowly moves outside the measurable spectral range at ambient pressure (i.e., in $\mathrm{SmTe}_{3}$ only its high-frequency tail is still observable). The remaining modes weakly disperse and progressively disappear when going from the La to the Dy compound along the rare-earth series.

Panels (b) and (c) of Fig. 1 display the Raman-scattering spectra of $\mathrm{LaTe}_{3}$ and $\mathrm{CeTe}_{3}$ under increasing and decreasing externally applied pressures. The spectral range covered within the DAC is limited at low frequencies at about $75 \mathrm{~cm}^{-1}$, while at ambient pressure it extends down to $65 \mathrm{~cm}^{-1}$. Therefore, the lowest zero-pressure mode $P 1$ cannot be clearly detected in the applied pressure experiment. As in the chemical-pressure case, all other modes slightly disperse and disappear upon applying pressure. This qualitative equivalence between chemical and applied pressures is also supported by the fact that the peaks in $\mathrm{LaTe}_{3}$ disappear at a slightly higher pressure than in $\mathrm{CeTe}_{3}$. The pressure dependence is fully reversible since upon decreasing pressure the modes reappear again.

The space group of the undistorted structure is $\mathrm{Cmcm}$ $\left(D_{2 h}^{17}\right)$ for all rare-earth tritellurides. From the occupied atomic positions ${ }^{18}$ and the factor-group analysis we determine the symmetry and multiplicity of the Raman-active phonons, ${ }^{19}$ namely, $4 A_{1 g}+4 B_{1 g}+4 B_{3 g}$. The corresponding Raman tensors imply that for our experimental configuration, in which both incident and scattered light are polarized parallel to the $a c$ crystal plane, only the $A_{1 g}$ symmetry phonons can be observed.
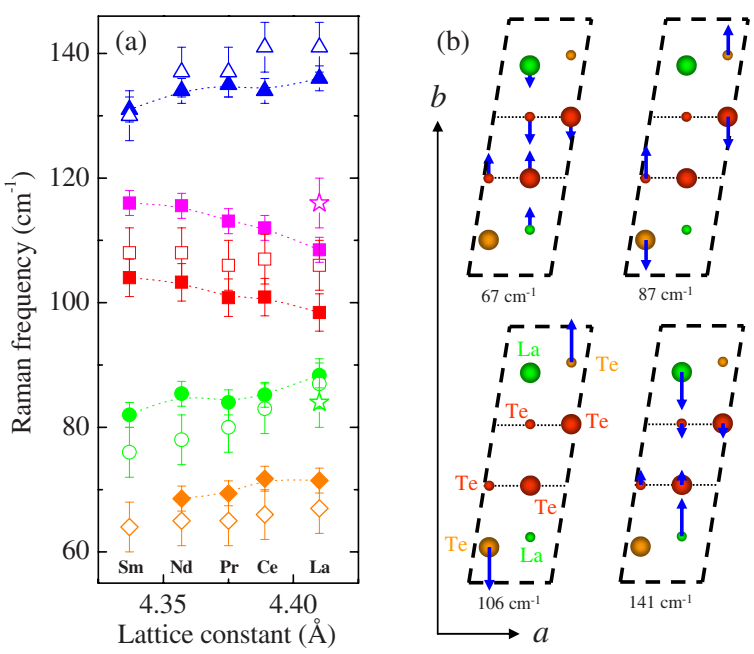

FIG. 2. (Color online) (a) Raman-active phonon frequencies of $R \mathrm{Te}_{3}$, obtained experimentally (solid symbols) and calculated for the undistorted structure (open symbols). The calculated extra peaks arising in the distorted structure (see text) are also shown with star symbols for $\mathrm{LaTe}_{3}$. The lattice-constant values are from Ref. 22. (b) Atomic displacements for the Raman-active $A_{1 g}$ vibrational modes in $\mathrm{LaTe}_{3}$. The figure shows the $a b$ plane, whereas large (small) spheres represent atoms having a positive (negative) $c$ coordinate. The dotted lines represent the Te planes. The primitive unit cell and crystal axes are also shown. Arrow lengths are proportional to the calculated displacements.

The vibrational modes at the $\Gamma$ point of the Brillouin zone for the undistorted structure at ambient pressure have been obtained from first principles using the $\mathrm{DMOL}^{3}$ code developed by one of us. ${ }^{20}$ First, the positions of the eight atoms in the primitive unit cell were optimized at the experimental lattice constants $a$ and $b,{ }^{21}$ obtained by averaging the values listed in the tables of Ref. 22. A frozen-phonon calculation then yielded the 24 sought-after frequencies. Only four of these correspond to the expected Raman-active modes with $A_{1 g}$ symmetry.

The frequencies of the calculated $A_{1 g}$ modes at $\Gamma$ for the $R \mathrm{Te}_{3}$ series are summarized in Fig. 2(a) along with the experimental values, while Fig. 2(b) pictures the $A_{1 g}$ lattice displacements of the undistorted structure, which, as predicted from the factor-group analysis, are along the $b$ axis. The agreement with the experimental findings is satisfactory. There is an obvious assignment of the calculated modes, for instance, at 67, 87, and $141 \mathrm{~cm}^{-1}$ for $\mathrm{LaTe}_{3}$, with the corresponding features in the measured spectra $(P 1, P 2$, and $P 5)$. The calculated mode at about $106 \mathrm{~cm}^{-1}$ lies between the experimentally observed modes $P 3$ and $P 4$ for $\mathrm{LaTe}_{3}$. This situation persists throughout the whole rare-earth series [Fig. 2(a)]. Our polarization-dependent measurements on $\mathrm{LaTe}_{3}$ [Fig. 3(a)] yield an angle-dependent intensity with a period of $90^{\circ}$ for the $P 4$ mode and $180^{\circ}$ for the adjacent peaks $P 2$ and $P 3 .^{23}$ Since a $180^{\circ}$ period is expected for the $A_{1 g}$ symmetry, the $P 4$ mode cannot be assigned within the undistorted structure.

In order to improve the mode assignment and to clarify the origin of the $P 4$ peak, we have, in a first step, computed the phonon dispersion in $\mathrm{LaTe}_{3}$ along the $\Gamma-Z$ direction of 

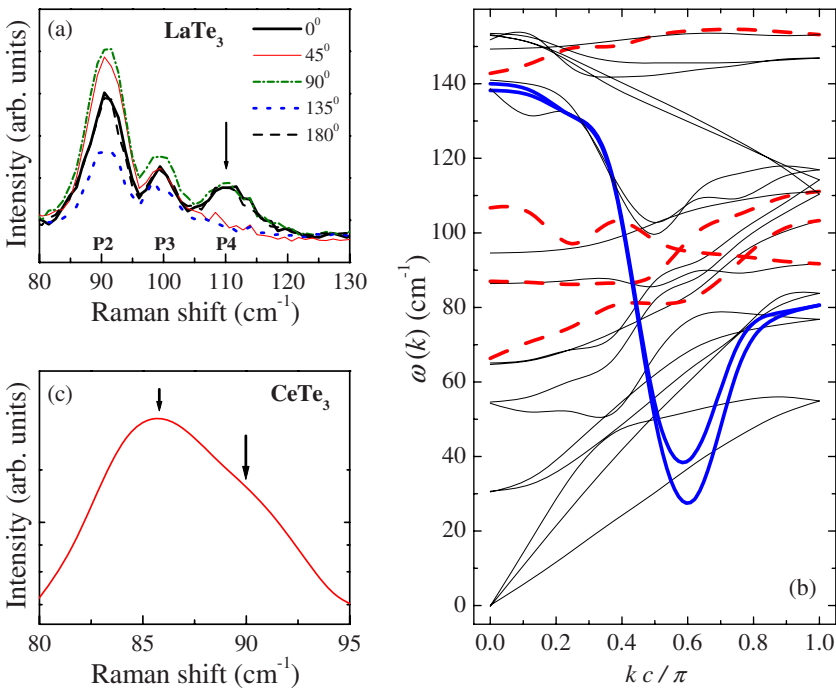

FIG. 3. (Color online) (a) Raman spectra of $\mathrm{LaTe}_{3}$ as a function of the polarization angle of the incident light. (b) Calculated phonon dispersion along the $c$ axis. (c) Enlargement of the $P 2$ peak in $\mathrm{CeTe}_{3}$ detailing its double-feature nature (logarithmic $y$ axis).

the Brillouin zone, using a supercell consisting of a 16-fold repetition along the $c$ axis of the primitive unit cell. This yields phonon frequencies at wave vectors $\left(0,0, q_{z}\right)$, where $q_{z}=\frac{n}{16} c^{*}$, with $c^{*}=\frac{2 \pi}{c}$, and $n$ between 0 and 8 . A symmetry constrained spline interpolation between these results is shown in Fig. 3(b) (similar results have been obtained along the $a$ axis). The dashed branches highlight the phonon dispersion for the modes with $A_{1 g}$ symmetry at $\Gamma$. The calculated phonon spectrum also shows a distinct Kohn anomaly at $q_{z}$ slightly below $0.3 c^{*}$ (thick lines), i.e., in the region expected from the electron-diffraction results ${ }^{9}$ and the angleresolved photoemission spectroscopy data on $\mathrm{CeTe}_{3}$ (Ref. 14). In a second step, we have generated a commensurate approximant to the distorted structure in the presence of the incommensurate CDW by repeating the calculation for a 14fold repetition of the primitive unit cell along the $c$ axis (in which case a true instability occurs at $q_{z}=\frac{2}{7} c^{*}$, i.e., the frequency of the soft phonon becomes imaginary), then moving the atoms along the eigenvectors of the soft phonon, and re-equilibrating their positions in the corresponding $(1 \times 1$ $\times 7)$ supercell. ${ }^{24}$ As a consequence of the lower symmetry, vibrational modes with $A_{1}$ and $B_{1}$ symmetry become Raman active. ${ }^{25}$ Although there are $56 A_{1}$ symmetry modes, their frequencies accumulate around those of the $A_{1 g}$ modes of the undistorted structure, suggesting that the distortion does not particularly affect the $\Gamma$-point vibrational energies. This is consistent with the fact that the phonon branches having $A_{1 g}$ symmetry at $\Gamma$ are weakly dispersing [Fig. 3(b)]. The main effect of the distortion is the appearance of $28 B_{1}$ modes, which accumulate around 84 and $116 \mathrm{~cm}^{-1}$. The latter frequency compares very nicely with the frequency of the $P 4$ peaks in our experiment. The second $B_{1}$ mode at about $84 \mathrm{~cm}^{-1}$ falls in the range of the $P 2$ peak. A closer look at the experimental data [Fig. 3(c) for $\left.\mathrm{CeTe}_{3}\right]$ indeed suggests that the $P 2$ peak may be a double feature.

An important result of our experimental investigation is

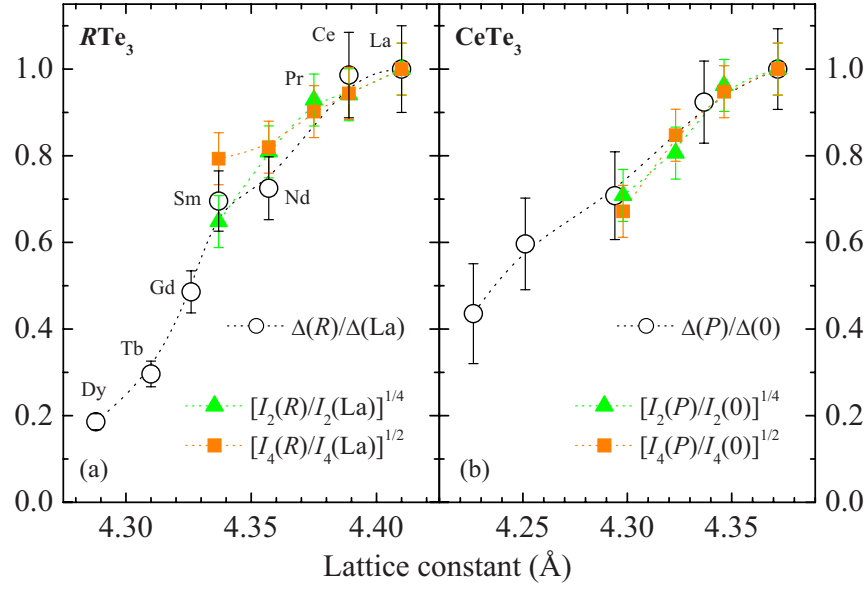

FIG. 4. (Color online) Comparison between the amplitude of the CDW gap (open circles) (Refs. 12 and 13) and the integrated intensities $(I)$ of the $P 2$ (full triangles) and $P 4$ (full squares) peaks, raised to $1 / 4$ and $1 / 2$, respectively (see text), as a function of the lattice constant (a) for the $R \mathrm{Te}_{3}$ series and (b) for $\mathrm{CeTe}_{3}$ under pressure. The pressure dependence of the lattice constant in $\mathrm{CeTe}_{3}$ was achieved following the procedure of Ref. 13. All quantities are normalized to their starting values.

the observation of a systematic decrease in the integrated intensity $(I)$ of the most prominent peaks $P 2$ and $P 4$ in the Raman spectra in Fig. 1 with pressure (i.e., the $B_{1}$ modes in the distorted structure of the CDW state), ${ }^{26}$ which bears a striking similarity with the behavior of the amplitude of the CDW gap $\Delta$ (i.e., the order parameter) upon compressing the lattice, as obtained from the optical conductivity. ${ }^{12,13}$ One could first argue that these modes disappear because of an enhancement of their width and a concomitant decrease in their apparent amplitude due to the increase in free carrier concentration upon compressing the lattice. ${ }^{12}$ The modes' width remains, however, almost constant so that this possibility is rather unlikely. Our optical data ${ }^{12}$ also allow us to exclude the possibility that the phonon modes disappear due to an increase in the absorption coefficient at the laser frequency with decreasing lattice constant. Figure 4 shows that the intensities of the $P 2$ and $P 4$ peaks scale fairly well with $\Delta^{4}$ and $\Delta^{2}$, respectively, ${ }^{27}$ suggestive of a coupling between the lattice vibrational modes and the CDW condensate. This is not at all surprising for the $P 4$ mode, as our calculations predict this peak only in the distorted structure. For the $P 2$ peak we should consider its two components, namely, the $A_{1 g}$ mode in the undistorted structure and the $B_{1}$ mode in the distorted one [Fig. 3(c)]. For the latter the intensity is obviously correlated with the $\mathrm{CDW}$, whereas for the former at $87 \mathrm{~cm}^{-1}$ the correlation can be explained by looking at the corresponding atomic displacements [Fig. 2(b)], which strongly distort the Te planes and therefore should couple to the CDW. ${ }^{28}$ Furthermore, the specific behavior $\left(I \sim \Delta^{q}, q=2\right.$ or 4 ) is consistent with theoretical predictions for the intensity in the distorted phase of originally silent modes, obtained from a group theoretical analysis in the framework of Landau's theory of second-order phase transitions. ${ }^{29}$

In summary, we have been able to draw a consistent picture of the Raman response of the CDW rare-earth tritellu- 
rides, by combining experimental observations and numerical simulations. In particular, we have provided clear evidence for the tight coupling between the CDW gap and the lattice degrees of freedom and have made a robust prediction for the Kohn anomaly inducing the CDW phase transition. Therefore, the easily tunable $R \mathrm{Te}_{3}$ series provides a rather unique playground for a systematic study of the mechanism leading to the formation of the CDW state.

The authors wish to thank S. L. Cooper, Z. X. Shen, and R. Hackl for fruitful discussions. This work was supported by the Swiss National Foundation for Scientific Research and by the NCCR MaNEP pool, as well as by the Department of Energy, Office of Basic Energy Sciences under Contract No. DE-AC02-76SF00515.
${ }^{1}$ R. Peierls, Quantum Theory of Solids (Clarendon, Oxford, 1955).

${ }^{2}$ M. D. Johannes and I. I. Mazin, Phys. Rev. B 77, 165135 (2008).

${ }^{3}$ G. Grüner, Density Waves in Solids (Addison-Wesley, Reading, MA, 1994).

${ }^{4}$ J. A. Wilson, F. J. DiSalvo, and S. Mahajan, Adv. Phys. 24, 117 (1975).

${ }^{5}$ J. Rouxel, in Crystal Chemistry and Properties of Materials with Quasi-One-Dimensional Structures, edited by J. Rouxel and D. Reidel (Springer, Dordrecht, 1986), pp. 1-26.

${ }^{6}$ T. Vuletić, B. Korin-Hamzić, T. Ivek, S. Tomić, B. Gorshunov, M. Dressel, and J. Akimitsu, Phys. Rep. 428, 169 (2006).

${ }^{7}$ See Sec. V of S. A. Kivelson, I. P. Bindloss, E. Fradkin, V. Oganesyan, J. M. Tranquada, A. Kapitulnik, and C. Howald, Rev. Mod. Phys. 75, 1201 (2003).

${ }^{8}$ H. Yao, J. A. Robertson, E. A. Kim, and S. A. Kivelson, Phys. Rev. B 74, 245126 (2006).

${ }^{9}$ E. DiMasi, M. C. Aronson, J. F. Mansfield, B. Foran, and S. Lee, Phys. Rev. B 52, 14516 (1995).

${ }^{10}$ N. Ru and I. R. Fisher, Phys. Rev. B 73, 033101 (2006).

${ }^{11}$ N. Ru, C. L. Condron, G. Y. Margulis, K. Y. Shin, J. Laverock, S. B. Dugdale, M. F. Toney, and I. R. Fisher, Phys. Rev. B 77, 035114 (2008).

${ }^{12}$ A. Sacchetti, L. Degiorgi, T. Giamarchi, N. Ru, and I. R. Fisher, Phys. Rev. B 74, 125115 (2006).

${ }^{13}$ A. Sacchetti, E. Arcangeletti, A. Perucchi, L. Baldassarre, P. Postorino, S. Lupi, N. Ru, I. R. Fisher, and L. Degiorgi, Phys. Rev. Lett. 98, 026401 (2007).

${ }^{14}$ V. Brouet, W. L. Yang, X. J. Zhou, Z. Hussain, N. Ru, K. Y. Shin, I. R. Fisher, and Z. X. Shen, Phys. Rev. Lett. 93, 126405 (2004).

${ }^{15}$ As the temperature dependence of the CDW gap is not known in $R \mathrm{Te}_{3}$, we choose pressure (both chemical and applied) as tunable variable.

${ }^{16}$ G. J. Piermarini, S. Block, and J. D. Barnett, J. Appl. Phys. 44, 5377 (1973).

${ }^{17}$ H. K. Mao, J. Xu, and P. M. Bell, J. Geophys. Res. 91, 4673 (1986).

${ }^{18}$ B. K. Norling and H. Steinfink, Inorg. Chem. 5, 1488 (1966).

${ }^{19}$ W. G. Fately, F. R. Dollish, N. T. McDevitt, and F. F. Bentley, Infrared and Raman Selection Rules for Molecular and Lattice Vibrations: The Correlation Method (Wiley, New York, 1972).
${ }^{20}$ The calculations were done with hardness-conserving semilocal pseudopotentials for all states below the $4 d$ shell for Te and the $5 s$ shell for the rare-earth atoms [B. Delley, Phys. Rev. B 66 , 155125 (2002)], and used the DNP basis set [B. Delley, J. Chem. Phys. 92, 508 (1990); 113, 7756 (2000)]; the $k$-space integrations have been performed with an unshifted $6 \times 2 \times 6$ $(2 \times 2 \times 6)$ mesh, which amounts to $40(16)$ symmetry unique $k$ points in the calculations for the primitive (super)cell. A thermal broadening of $1 \mathrm{mHartree}$ has been used. The total energy has been modified with the entropy term proposed by M. Weinert and J. W. Davenport, Phys. Rev. B 45, 13709 (1992) to make the energy functional variational.

${ }^{21}$ An orthorhombic pseudotetragonal $(a=c)$ structure was considered, as the small deviations between $a$ and $c$ were found to be insignificant in an earlier high-precision calculation of the Fermi surface [J. Laverock, S. B. Dugdale, Z. Major, M. A. Alam, N. Ru, I. R. Fisher, G. Santi, and E. Bruno, Phys. Rev. B 71, 085114 (2005)].

${ }^{22}$ P. Villars and L. D. Calvert, Pearsons Handbook of Crystallographic Data for Intermetallic Phases (American Society for Metals, Metals Park, OH, 1991).

${ }^{23}$ We also collected data (not shown here) with the scattered light polarization orthogonal to the incident one and obtained results fully consistent with those of the parallel polarization [Fig. 3(a)].

${ }^{24}$ C. Malliakas, S. J. L. Billinge, H. J. Kim, and M. G. Kanatzidis, J. Am. Chem. Soc. 127, 6510 (2005).

${ }^{25}$ The unit cell in the distorted structure is not centrosymmetric anymore, so that the $A_{1 g}$ symmetry turns into $A_{1}$. The period of the angle-dependent intensity is $180^{\circ}\left(90^{\circ}\right)$ for the $A_{1}\left(B_{1}\right)$ symmetry.

${ }^{26}$ The sudden disappearance of the modes in the Raman spectra of Gd and Dy tritellurides [Fig. 1(a)] could also originate from the fact that the data were collected at $300 \mathrm{~K}$, i.e., just slightly below their own $T_{\text {CDW }}$ (Ref. 11).

${ }^{27}$ The behavior of the weak $P 3$ and $P 5$ peaks is less well defined, while the intensity of the $P 1$ peak, which is only partially seen in the chemical-pressure series, does not seem to change much.

${ }^{28}$ M. J. Rice, Phys. Rev. Lett. 37, 36 (1976); Solid State Commun. 25, 1083 (1978).

${ }^{29}$ J. Petzelt and V. Dvořák, J. Phys. C 9, 1571 (1976). 